\title{
Geoprocessamento e Sensoriamento remoto aplicados no levantamento e mapeamento da degradação vegetal entre 1987 e 2010 no município de Iati-PE
}

Geoprocessiong and Remote Sensing applied in the survey and mapping of vegetation degradation between 1987-2010 in the country of Iati-PE

\author{
ALEXANDRE ${ }^{1}$, F. S.; RAMOS ${ }^{1}$, P. S.; DEUS ${ }^{1}$, R. A. S. G.; $\operatorname{SILVA}^{1}$, S. A.; \\ GOMES ${ }^{1}$, D. D. M. \\ fnando257@gmail.com
}

\begin{abstract}
Resumo
Com a demanda global de recursos naturais para os mais diversos fins, ficou evidente a necessidade de se ter um planejamento prévio para a sua retirada, para que assim o meio não seja alterado de forma drástica, e um dos principais reguladores desse meio é a vegetação, que desempenha grande papel na hidrologia, geologia, geomorfologia, pedologia e climatologia. Para tanto é de grande importância que se faça um estudo da mesma, analisando as mudanças ocorridas, seja pela ação humana ou natural. Este trabalho visa mapear a degradação da vegetação no município de Iati-PE nos anos de 1987, 1994, 2000, 2004 e 2010, utilizando-se do Geoprocessamento e do Sensoriamento remoto para obter resultados mais fidedignos.
\end{abstract}

Palavras-chave: Degradação vegetal, Iati, SIG.

\begin{abstract}
With global demand for natural resources for various purposes, it was evident the need to have prior planning for withdrawal, so that the medium is not changed drastically, and one of the main regulators of this medium is the vegetation which plays big role in hydrology, geology, geomorphology, soil science and climatology. Therefore it is of great importance to make a study of it, analyzing the changes occurred, whether by human or natural action. This work aims to map the vegetation degradation in Iati-PE municipality in 1987, 1994, 2000, 2004 and 2010, using the GIS and Remote Sensing for more reliable results.
\end{abstract}

Keywords: plant degradation, Iati, GIS.

\section{INTRODUÇÃO}

Com a demanda global de recursos naturais para os mais diversos fins, ficou evidente a necessidade de se ter um planejamento prévio para a retirada desses recursos, para que não aconteça um desequilíbrio no ecossistema. Um dos recursos mais extraído pelo homem é a madeira, que controla a dinâmica da troca de energia e matéria, através da hidrologia, geologia, geomorfologia, pedologia e climatologia entre outras (YI et al. 2012).

Com tal importância é essencial que se faça estudos acerca da sua degradação, pois assim obteremos os dados de como se deu as alterações no local de estudo, entendendo melhor toda a dinâmica local e como se dá o uso e ocupação do solo. Pode-se detectar os níveis de mudança da cobertura vegetal através de métodos e técnicas, utilizando os Índices de Vegetação de duas ou mais datas (TRINCART, 1977). Um dos Índices mais usados e que proporciona um melhor resultado é o NDVI (Normalized Difference Vegetation Index), que se

\footnotetext{
${ }^{1}$ Fernando da Silva Alexandre, Geografia/LabGeo, Universidade de Pernambuco, Garanhuns-PE, Brasil

${ }^{1}$ Renilson Pinto da Silva Ramos, Geografia/LabGeo, Universidade de Pernambuco, Garanhuns-PE, Brasil

${ }^{1}$ Rodolfo Alexandre da Silva Gomes de Deus, Geografia/LabGeo, Universidade de Pernambuco, Garanhuns-PE, Brasil

${ }^{1}$ Suzana de Araújo Silva, Geografia/LabGeo, Universidade de Pernambuco, Garanhuns-PE, Brasil

${ }^{I}$ Daniel Dantas Moreira Gomes, Geografia/LabGeo, Universidade de Pernambuco, Garanhuns-PE, Brasil
} 
utiliza dos níveis de reflectância das folhas verdes sadias a partir do comprimento de cada onda do espectro eletromagnético refletido, permitindo identificar cada porção de determinado comprimento de onda (PONZONI et al. 2012).

O melhor meio de se fazer um estudo de uma área de tamanha proporção é o Sensoriamento Remoto (SR) em conjunto com um Sistema de Informação Geográfica (SIG), utilizando-se do Geoprocessamento para incumbir tudo em uma base de dados sólida e versátil. Neste estudo foi decidido trabalhar com cenas do sensor orbital Landsat 5, pois o mesmo ficou um grande período em orbita, permitindo a captura de dados com grande intervalo de tempo, porém foram coletas imagens para este estudo apenas entre os meses de setembro a fevereiro, o que corresponde a época mais seca do ano, afim de que se obtenha um melhor resultado na classificação (ROSA, 2009)

O Sensoriamento Remoto (SR) pode ser definido como a técnica capaz de obter dados sem que o mesmo esteja em contato com o alvo, através da Radiação eletromagnética (REM) refletida pelas componentes da superfície, onde são captadas pelos sensores orbitais, possibilitando por meio do comprimento das ondas identificar cada objeto e o seu nível de reflectância (FITZ, 2013).

Com o intuito de observar as mudanças ocasionadas pela degradação da vegetação, decidiu-se classificar o mapeamento em 5 classes de acordo com Yengoh et al. (2015) em: conservada, parcialmente conservada, degradada, fortemente degradada e solo exposto. $\mathrm{O}$ principal objeto de estudo é mapear e comparar a degradação da vegetação do município de Iati-PE nas datas, 17/01/1987, 09/02/1994, 06/12/2000, 10/12/2004 e 29/09/2010.

O município de Iati, localiza-se entre os paralelos 853'17"/ 9²2’54" S e os meridianos 3703'23"/ 36 46'04” W no Datum WGS 84, na microrregião geográfica de Garanhuns, que se situa no Agreste de Pernambuco. O município possui uma área de 635,465 $\mathrm{Km}^{2}$ de área territorial (figura 1). Com seu Bioma classificado em Caatinga, com a vegetação predominante de Florestas Subcaducifólica (Mata úmida) e Caducifólica. 


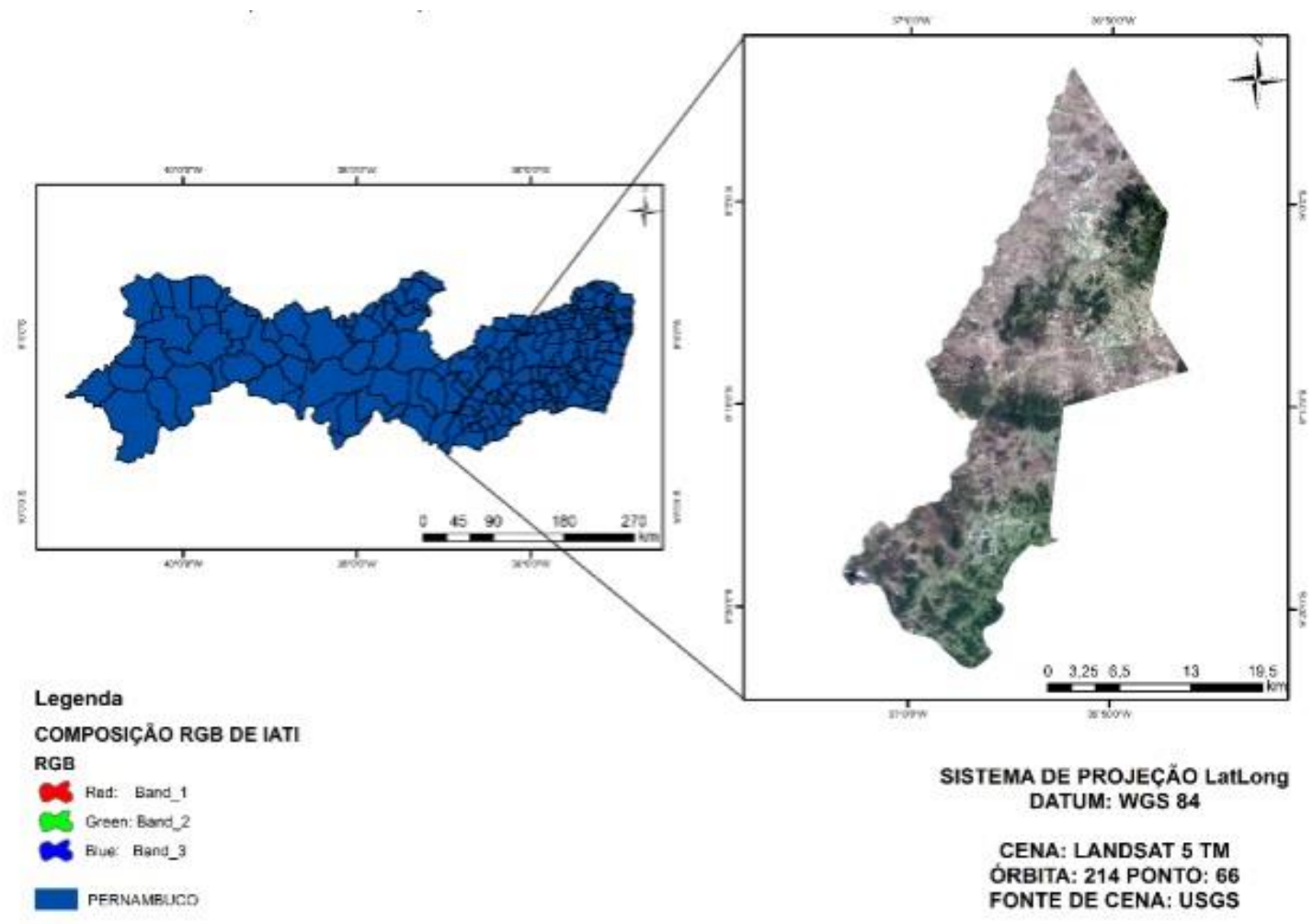

Figura 01. Mapa de localização do objeto de estudo. Fonte: Elaborado pelos autores

\section{METODOLOGIA}

\subsection{AQUISIÇÃO DAS IMAGENS ORBITAIS}

$\mathrm{Na}$ busca de melhores resultados e mais fidedignos com a realidade, buscou-se fazer este estudo com imagens do satélite Landsat 5 com o sensor TM (Thematic Mapper) de 8 bits (256 níveis de cinza), com uma resolução espacial de 30x30 metros, nas datas 17/01/1987, 09/02/1994, 06/12/2000, 10/12/2004 e 29/09/2010, disponibilizadas no site da USGS (United States Geological Survey), www.usgs.com.

\subsection{MATERIAIS}

Inicialmente fez-se o levantamento bibliográfico, material cartográfico e imagens orbitais. Foram consultados, livros, artigos, teses, monografias, dissertações e trabalhos técnicos, para dar fomento teórico a pesquisa.

Foram utilizadas cenas obtidas a partir Landsat 5- TM que são dados raster, utilizouse unicamente as bandas 4 e 5 que corresponde a banda do vermelho e do infravermelho respectivamente. Foram utilizadas as cenas da orbita 214 e do ponto 66, em que foi feita a 
correção geometria e radiométrica através do software ArcGIS ${ }^{\circledR} 10.3$, licenciado por "licença ArcGIS for Personal Use".

Os dados cartográficos foram armazenados no $\operatorname{ArcGIS}^{\circledR} 10.3$ possibilitando o armazenamento e o gerenciamento dos dados em um banco de dados criado no próprio software, facilitando trabalhar as cenas e outros dados.

\subsection{PROCESSAMENTO DOS DADOS}

Todo o processamento dos dados foi feito no ArcGIS 10.3 as cenas obtidas a partir do Landsat 5 foram recortadas nos limites do município com base em um shapefile obtido no site do IBGE, no Arctoolbox, Data management Toll, pasta Raster, subpasta Raster processing, ferramenta Clip.

Seguiu-se do cálculo do NDVI (figura 2), no Arctoolbox Spatial Analyst Toll, Map Algebra, ferramenta Raster Calculator. No cálculo do NDVI é feito a subtração das bandas espectrais do infravermelho próximo e a banda do vermelho dividido pela soma das duas, feito isso nas cinco datas, seguida da classificação supervisionada (máxima verossimilhança) e posteriormente foi feito o cálculo para saber a área que cada classe ocupa.

Figura02: Equação do NDVI. Fonte: Elaborado pelos autores

$$
\mathrm{NDVI}=\frac{\mathrm{IVP}-\mathrm{V}}{\mathrm{IVP}+\mathrm{V}}
$$

Onde:

IVP= infravermelho próximo

$\mathrm{V}=$ Vermelho

Posteriormente é feita a coleta de amostras de cada classe para a melhor classificação, cria-se um novo shapefile, e neste se coleta as amostras, editando sua tabela nomeando cada classe, no total foram coletadas cerca de 150 amostras, sendo 30 em cada uma das classes.

Logo em seguida utilizou-se o Arctoolbox Spatial Analyst Toll, pasta Multivariate, subpasta ferramenta Create Signatures. Nessa etapa se faz a junção da imagem Raster com os dados do Shapefile, a partir de cálculos ele apresenta uma Signature com os padrões espectrais, criando um arquivo GSG. Seguindo-se da utilização da ferramenta Maximum Likelihood 
Classification, na qual o programa se utiliza da cena e da Signature, obtendo como resultado a imagem já classificada, de acordo com cada classe.

\subsection{SEQUÊNCIA METODOLÓGICA}

Para melhor explanar a sequência metodológica foi feito um fluxograma com cada etapa da elaboração do trabalho, dando destaque aos principais pontos do desenvolvimento, pode-se observar o procedimento metodológico na da figura 3.

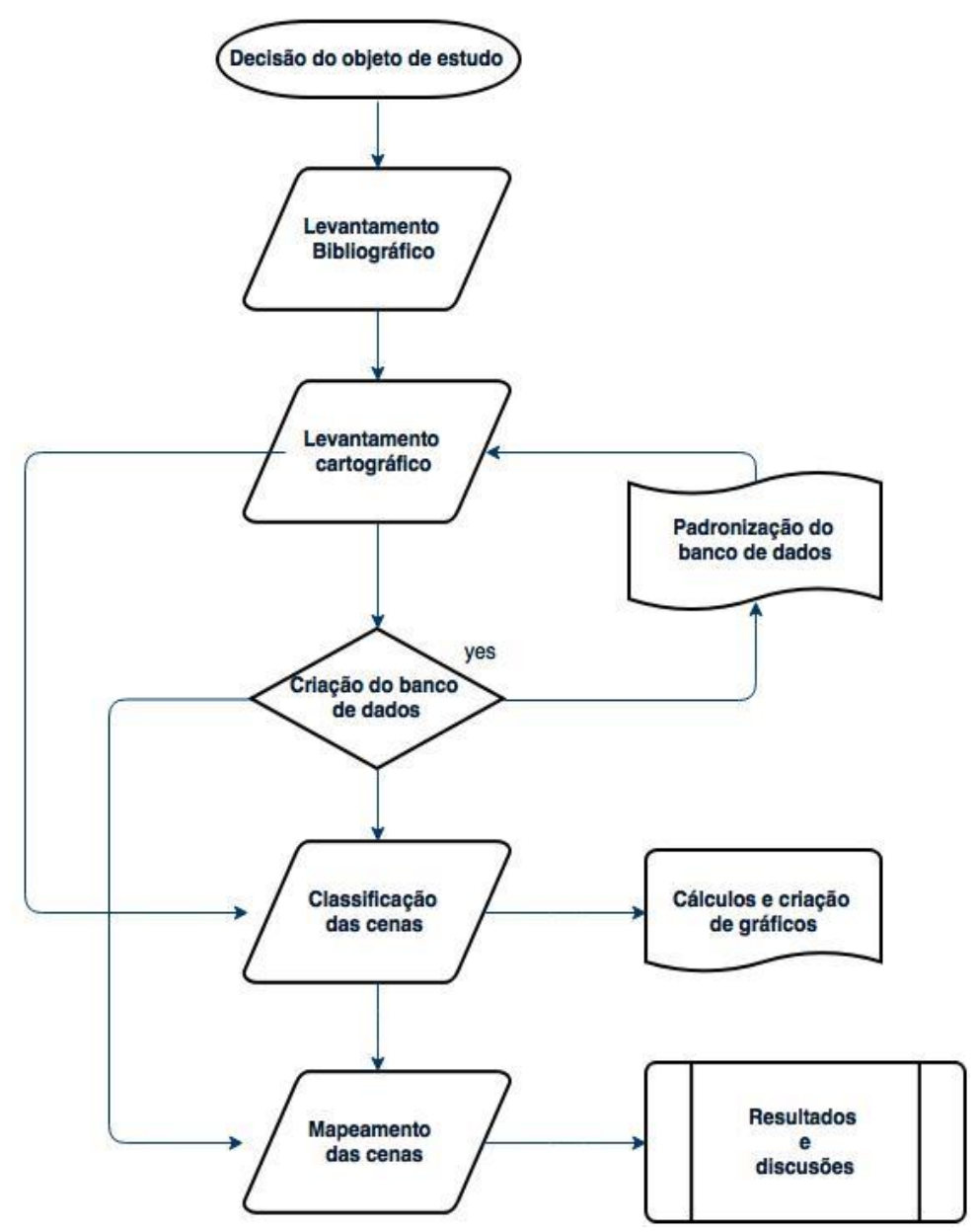

Figura 03. Fluxograma com as etapas metodológicas. Fonte: Elaborado pelos autores

Levou-se em consideração as peculiaridades locais, as espécies dominantes na região fazem parte da Caatinga: carnaúba (Copernicia prunifera), catingueira (Caesalpínia bracteosa), pinhão bravo (Jatropha molissima), juazeiro (Ziziphus juazeiro), oiticica (Licania rígida), marmeleiro preto (Croton sonderianus), jurema preta (Mimosa tenuifolia), jurema branca (Píptadenia stipulacea), velame (Croton sp), coronha (Acácia farnesiana), jucá (Ceasalpínia ferrea), sabiá (Mimosa caesalpiniaefolia Benthan), mofumbo (Combretum 
leprosum), paubranco (Auxemma oncocalyx) e as cactáceas: xiquexique (Ploceurus gounelli), mandacaru (Cereus jamacaru), facheiro (Pilosocereus pachycladus), entre outras (FERNANDES et al 1990 e 1998, citado por GOMES et al. 2011).

\section{RESULTADOS E DISCUSSÕES}

Após a classificação supervisionada foi possível identificar e mapear a degradação da vegetação espacialmente e temporalmente comprovando a grande validade do NDVI, mostrando que o Sensoriamento Remoto é uma técnica poderosa, que juntamente com o Geoprocessamento permite o estudo de um dossel vegetal de forma rápido e com boa qualidade nos dados obtidos.

O município de Iati-PE, mostrou grande variação em sua vegetação nas cinco datas, de 1987 a 2010, mostrando assim a necessidade da elaboração de mapas temáticos de cada data, tornando assim a visualização dos dados mais fácil (RAMOS et al. 2014).

A vegetação conservada obteve nos anos de 1987, 1994, 2000, 2004 e 2010 respectivamente: $11,192 \mathrm{Km}^{2}, 22,591 \mathrm{Km}^{2}, 3,600 \mathrm{Km}^{2}, 3,954 \mathrm{Km}^{2}, 9,670 \mathrm{Km}^{2}$, a vegetação parcialmente conservada obteve nos anos de 1987, 1994, 2000, 2004 e 2010 respectivamente: $263,003 \mathrm{Km}^{2}, 147,820 \mathrm{Km}^{2}, 70,748 \mathrm{Km}^{2}, 11,253 \mathrm{Km}^{2}, 96,140 \mathrm{Km}^{2}$, a vegetação degradada obteve nos anos de 1987, 1994, 2000, 2004 e 2010 respectivamente: 291,283 Km² 336,023 $\mathrm{Km}^{2}, 521,568 \mathrm{Km}^{2}, 268,366 \mathrm{Km}^{2}, 268,374 \mathrm{Km}^{2}$, a vegetação fortemente degradada obteve nos anos de 1987, 1994, 2000, 2004 e 2010 respectivamente: 62,620 Km², 123,266 Km², $33,926 \mathrm{Km}^{2}, 120,989 \mathrm{Km}^{2}, 230,260 \mathrm{Km}^{2}$, o solo exposto obteve nos anos de 1987, 1994, 2000, 2004 e 2010 respectivamente: 6,960 $\mathrm{Km}^{2}, 5,648 \mathrm{Km}^{2}, 5,528 \mathrm{Km}^{2}, 230,780 \mathrm{Km}^{2}$, $30,909 \mathrm{Km}^{2}$.

Pode-se observar nas figuras 4, 5, 6, 7 e 8 o mapeamento da vegetação do município de Iati nos anos de 1987, 1994, 2000, 2004 e 2010 respectivamente, e no gráfico 1 se percebe a grande discrepância em cada classe nas cinco datas. 


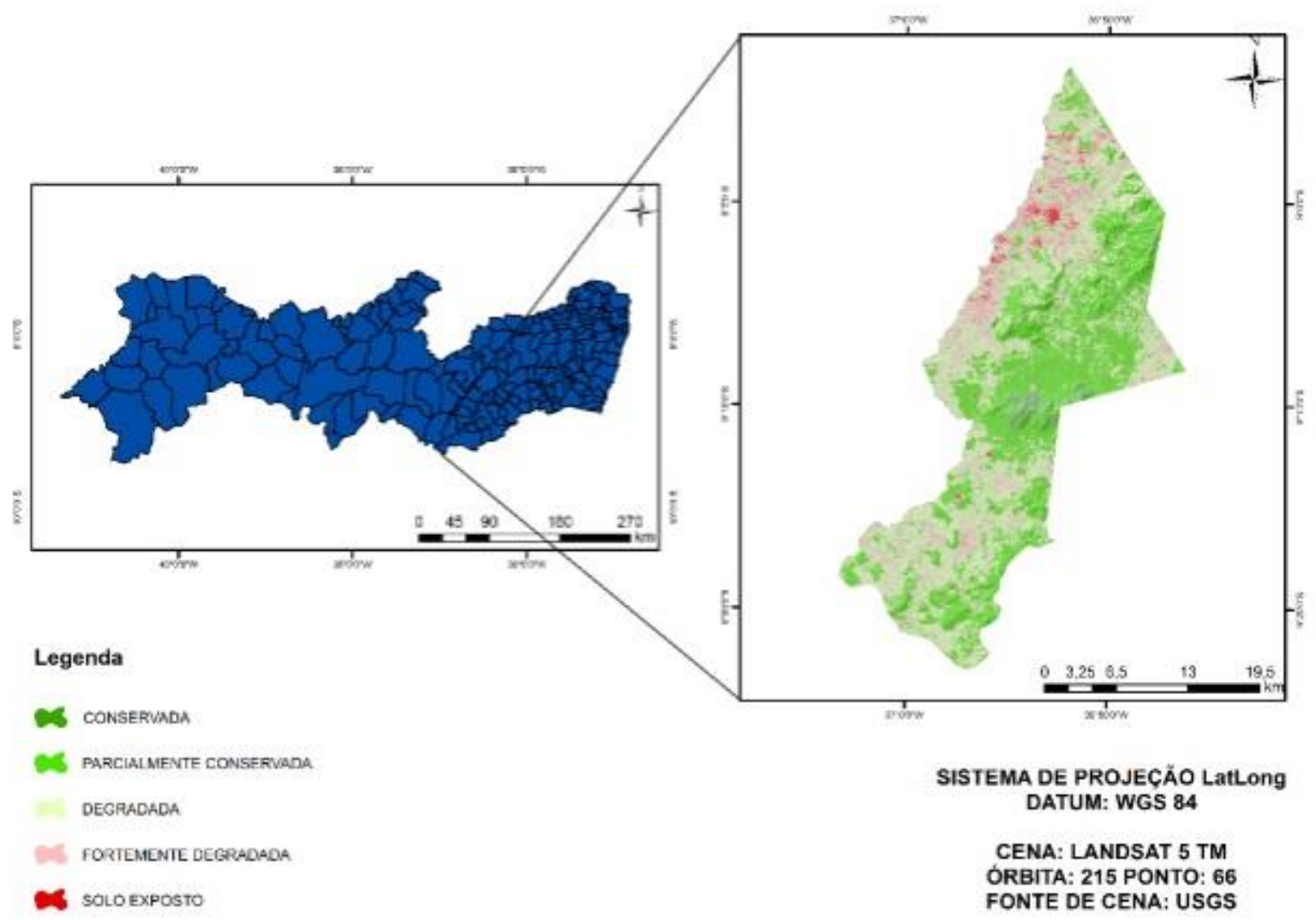

Figura 04. Mapa da cobertura vegetal no ano de 1987. Fonte: Elaborado pelos autores.

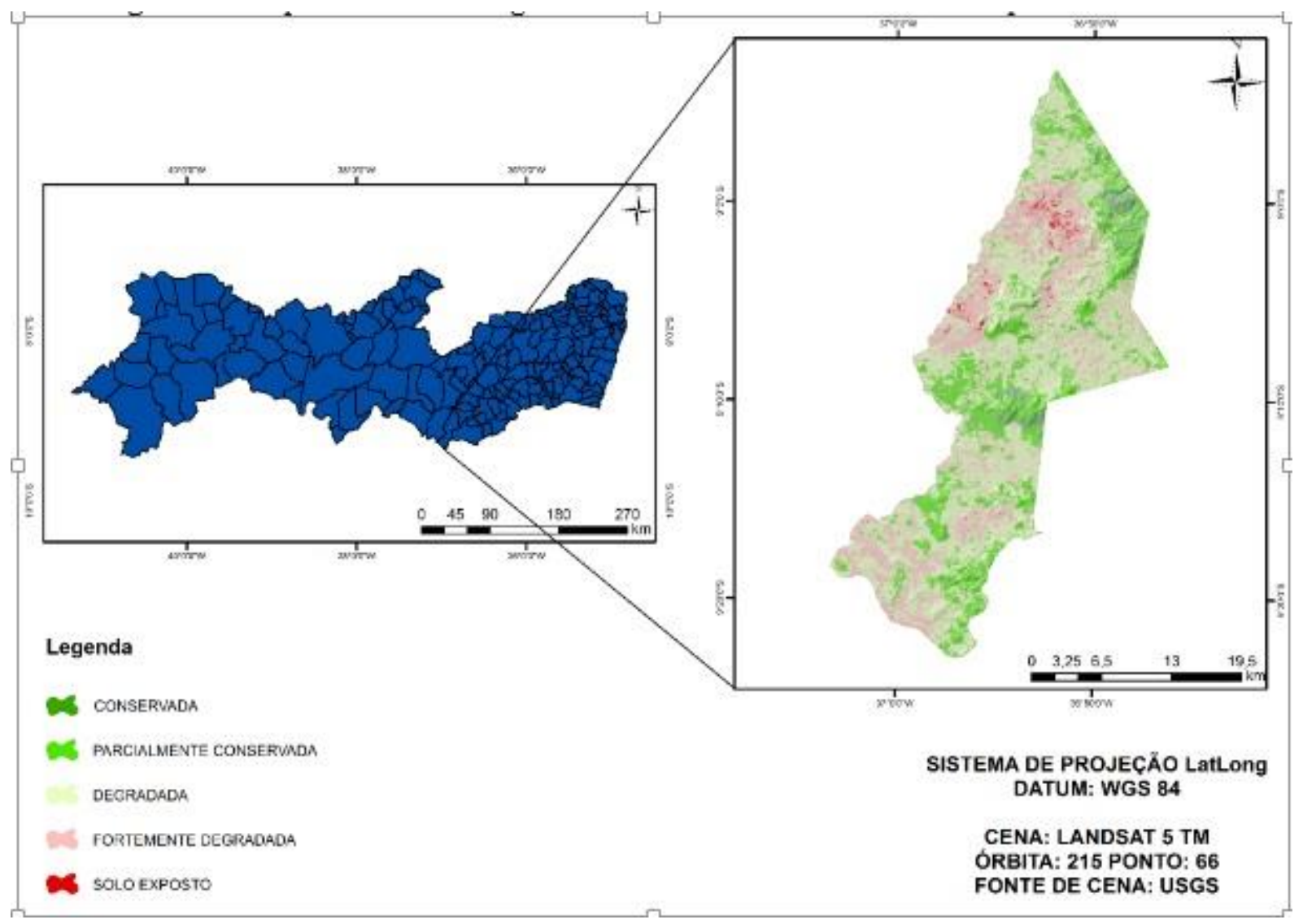

Figura 05. Mapa da cobertura vegetal no ano de 1994. Fonte: Elaborado pelos autores. 


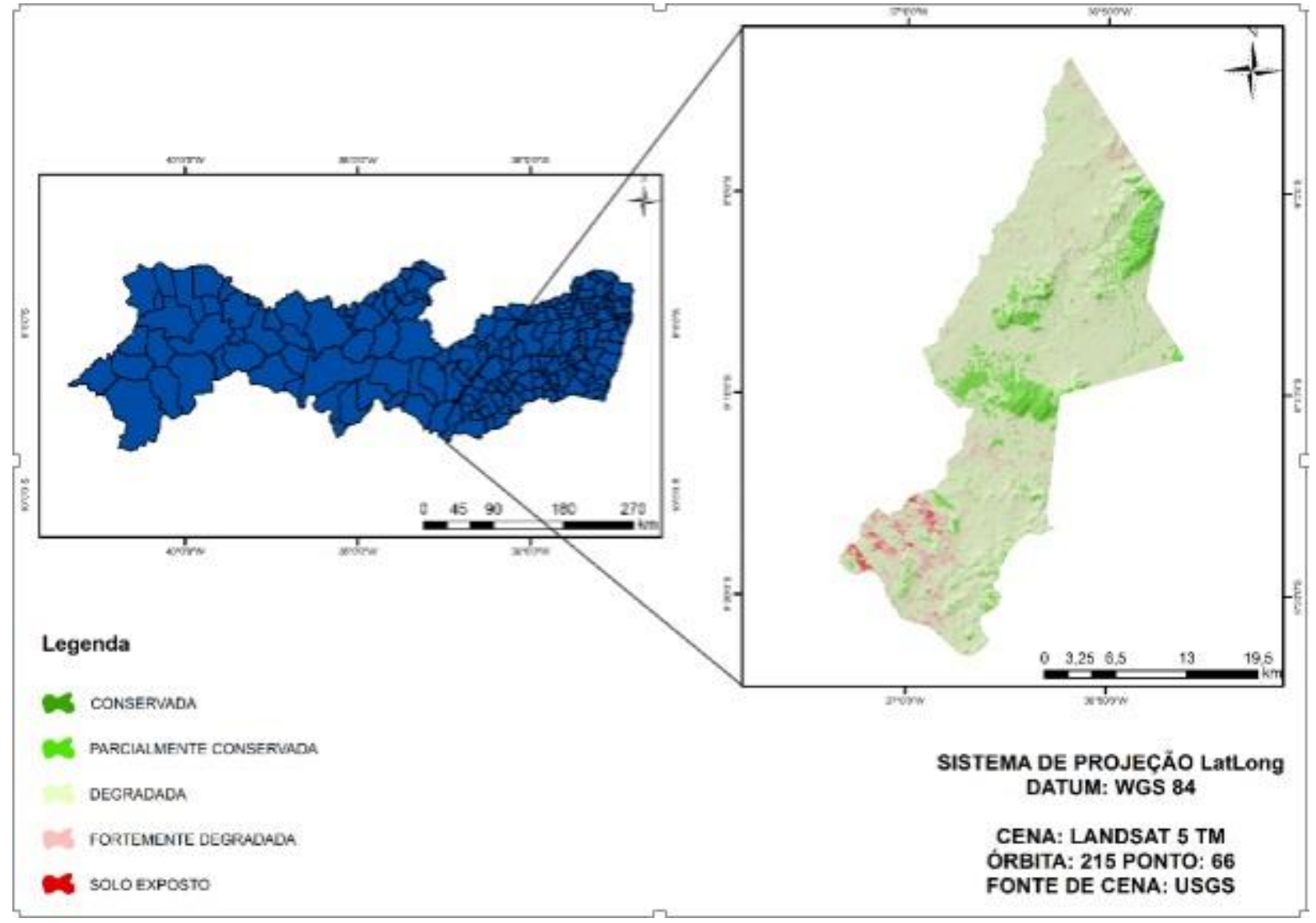

Figura 06. Mapa da cobertura vegetal no ano de 2000. Fonte: Elaborado pelos autores.

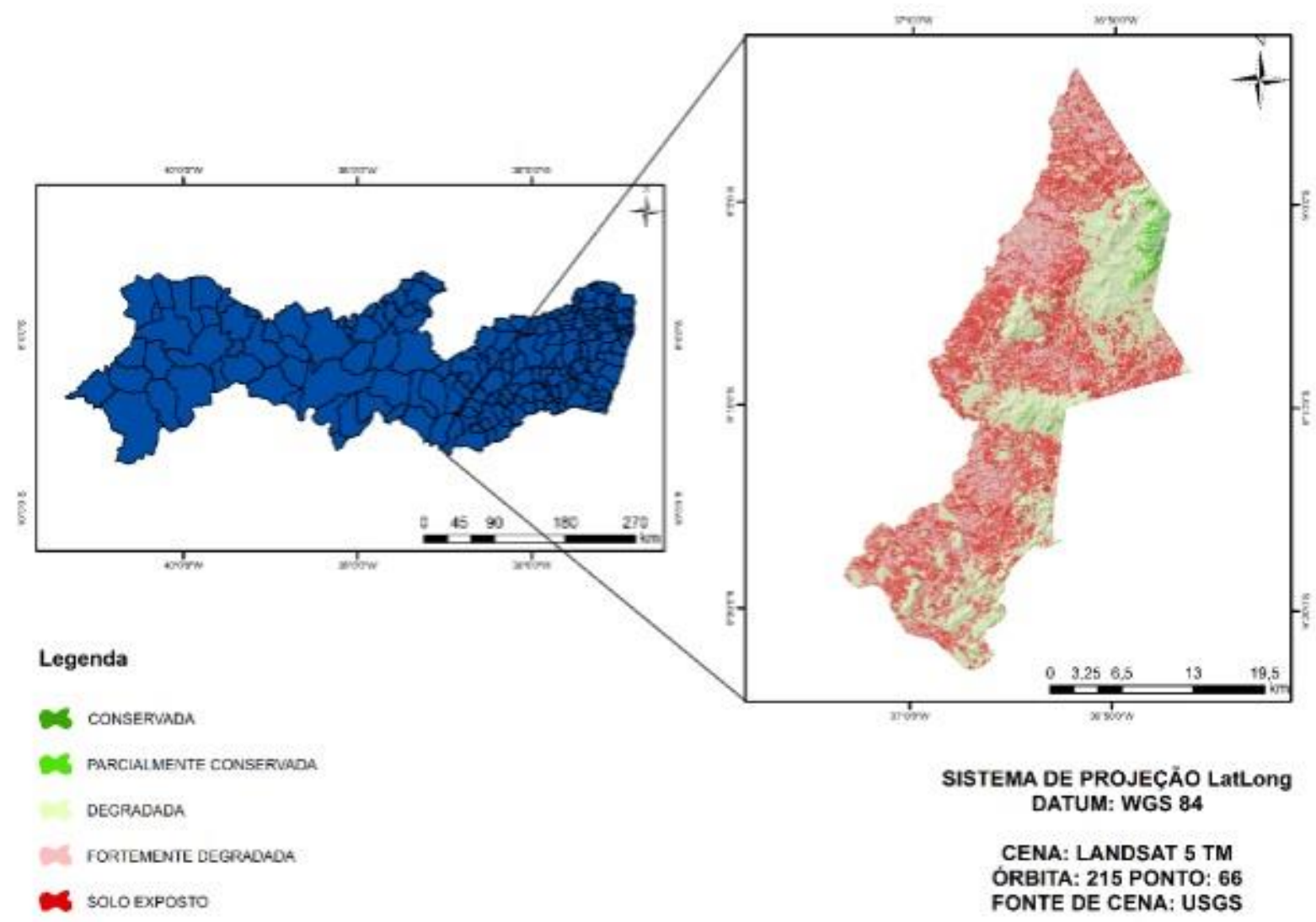

Figura 07. Mapa da cobertura vegetal no ano de 2004. Fonte: Elaborado pelos autores. 


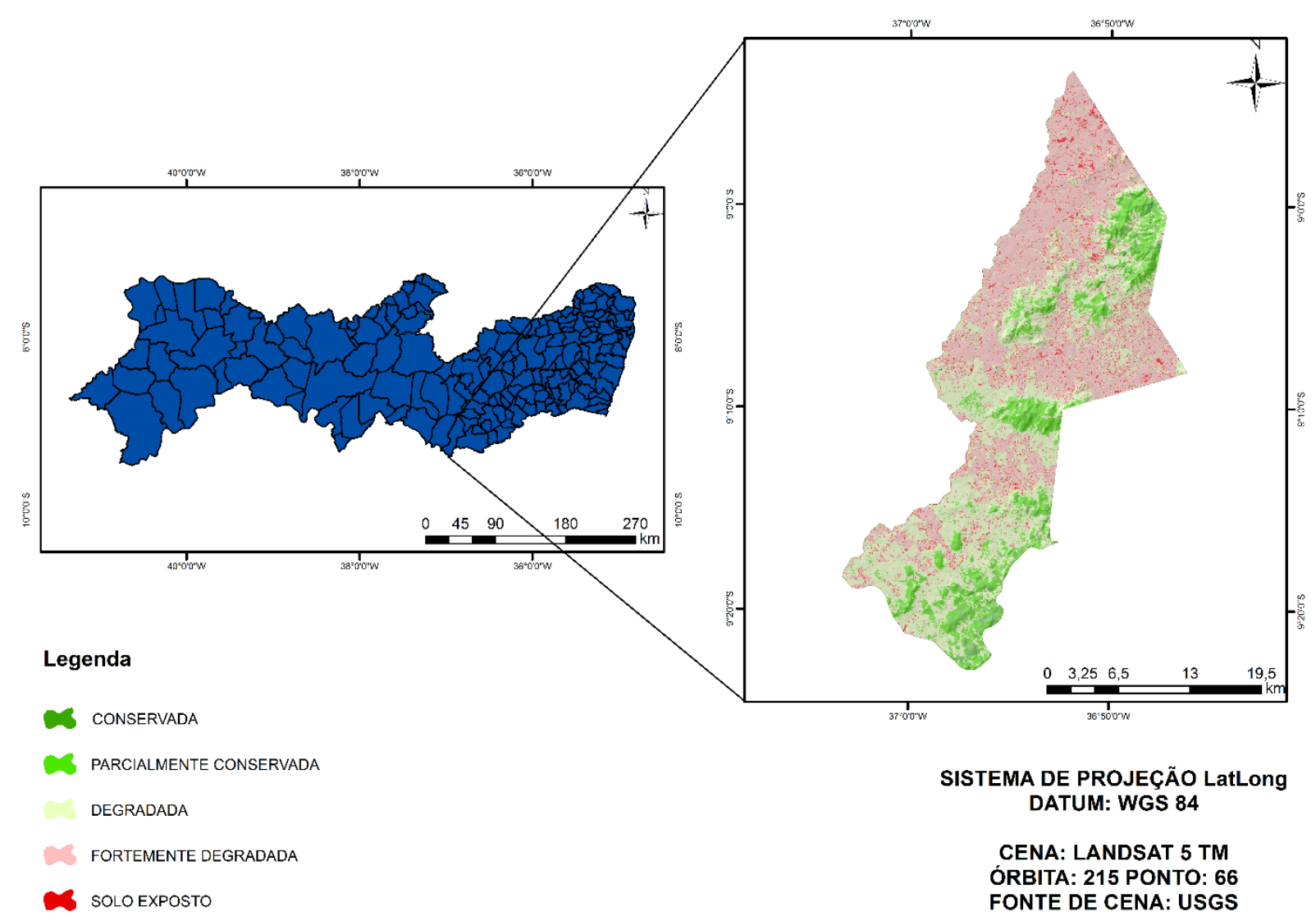

Figura 08. Mapa da cobertura vegetal no ano de 2010. Fonte: Elaborado pelos autores.

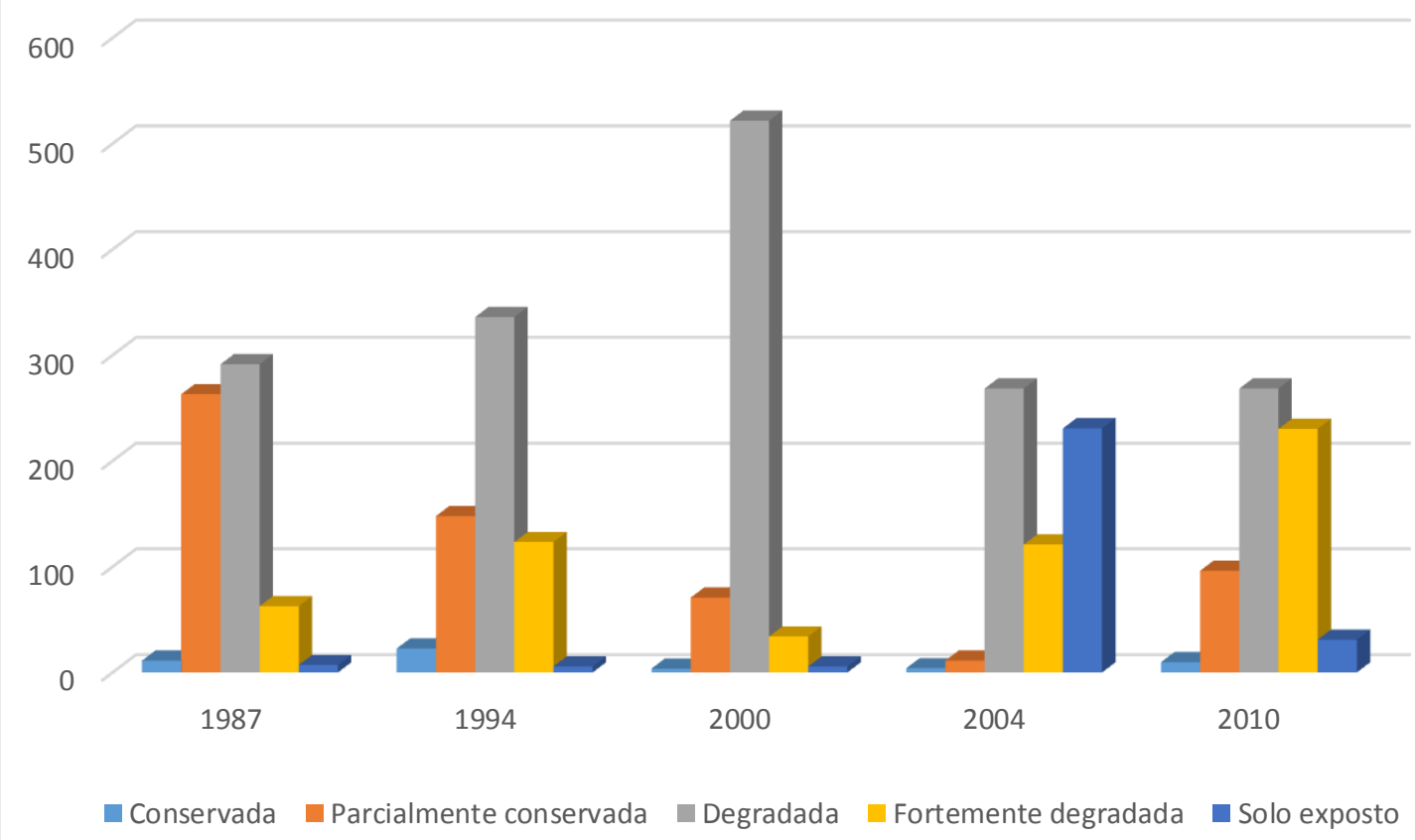

Gráfico 01. Apresenta a área que cada classe ocupa. Fonte: Elaborado pelos autores. 


\section{CONSIDERAÇÕES FINAIS}

O SR juntamente com o Geoprocessamento mostrou a grande versatilidade e a praticidade na elaboração e levantamento de dados relacionados a vegetação, permitindo que houvesse o levantamento e o mapeamento da degradação vegetal por causas naturais e antrópicas desde 1987 a 2010 . Permitiu o planejamento e o gerenciamento do território pelos gestores públicos. Pode-se observar nos quadros1 e 2 a disparidade da área e da porcentagem obtida por cada classe nos anos de 1987, 1994, 2000, 2004 e 2010.

\begin{tabular}{|c|c|c|c|c|c|}
\hline Classes & 1987 & 1994 & 2000 & 2004 & 2010 \\
\hline Conservada & 11,192 & 22,591 & 3,600 & 3,954 & 9,670 \\
& $\mathrm{Km}^{2}$ & $\mathrm{Km}^{2}$ & $\mathrm{Km}^{2}$ & $\mathrm{Km}^{2}$ & $\mathrm{Km}^{2}$ \\
\hline Parcialmente & 263,003 & 147,820 & 70,747 & 11,226 & 96,140 \\
\hline conservada & $\mathrm{Km}^{2}$ & $\mathrm{Km}^{2}$ & $\mathrm{Km}^{2}$ & $\mathrm{Km}^{2}$ & $\mathrm{Km}^{2}$ \\
\hline Degradada & 291,282 & 336,023 & 521,568 & 268,466 & 268,375 \\
\hline Fortemente & $\mathrm{Km}^{2}$ & $\mathrm{Km}^{2}$ & $\mathrm{Km}^{2}$ & $\mathrm{Km}^{2}$ & $\mathrm{Km}^{2}$ \\
\hline degradada & 62.620 & 123,366 & 33,926 & 120,989 & 231,380 \\
\hline Solo Exposto & $\mathrm{Km}^{2}$ & $\mathrm{Km}^{2}$ & $\mathrm{Km}^{2}$ & $\mathrm{Km}^{2}$ & $\mathrm{Km}^{2}$ \\
& 6,960 & 5,643 & 5,528 & 230,780 & 30,909 \\
\hline TOTAL & $\mathrm{Km}^{2}$ & $\mathrm{Km}^{2}$ & $\mathrm{Km}^{2}$ & $\mathrm{Km}^{2}$ & $\mathrm{Km}^{2}$ \\
\hline & $\mathbf{6 3 5 , 4 6 5}$ & $\mathbf{6 3 5 , 4 6 5}$ & $\mathbf{6 3 5 , 4 6 5}$ & $\mathbf{6 3 5 , 4 6 5}$ & $\mathbf{6 3 5 , 4 6 5}$ \\
\hline
\end{tabular}

Quadro 1: Análise comparativa das áreas obtidas em cada classe através do NDVI. Fonte: Elaborado pelos autores

\begin{tabular}{|c|c|c|c|c|c|}
\hline Classes & $\mathbf{1 9 8 7}$ & $\mathbf{1 9 9 4}$ & $\mathbf{2 0 0 0}$ & $\mathbf{2 0 0 4}$ & $\mathbf{2 0 1 0}$ \\
\hline Conservada & $0,17 \%$ & $3,55 \%$ & $0,57 \%$ & $0,62 \%$ & $1,52 \%$ \\
\hline $\begin{array}{c}\text { Parcialmente } \\
\text { conservada }\end{array}$ & $41,38 \%$ & $23,26 \%$ & $11,15 \%$ & $1,76 \%$ & $15,13 \%$ \\
\hline Degradada & $46,83 \%$ & $52,51 \%$ & $82,07 \%$ & $42,27 \%$ & $42,13 \%$ \\
\hline $\begin{array}{c}\text { Fortemente } \\
\text { degradada }\end{array}$ & $9,85 \%$ & $19,40 \%$ & $5,34 \%$ & $19,04 \%$ & $36,41 \%$ \\
\hline Solo Exposto & $1,77 \%$ & $0,88 \%$ & $0,87 \%$ & $33,31 \%$ & $4,81 \%$ \\
\hline TOTAL & $\mathbf{1 0 0} \%$ & $\mathbf{1 0 0} \%$ & $\mathbf{1 0 0} \%$ & $\mathbf{1 0 0} \%$ & $\mathbf{1 0 0} \%$ \\
\hline
\end{tabular}

Quadro 2: Análise comparativa das percentagens obtidas em cada classe através do NDVI. Fonte: Elaborado pelos autores

\section{REFERÊNCIAS}

FITZ, P. R. Geoprocessamento sem complicação. 3.ed. revisada e ampliada. São Paulo. 2013. 26p.

YENGOH, G. T. DENT, D. OLSSON, L. TENBERG, A. E. TUCKER, C.J Use of the Normalized Diferrence Vegetation Index (NDVI) to Asses Lan Degradation at Multiple 
Scales: Curremt Status, Future Trends, and Practical Cosiderations. Springer, New York. 2015.

GOMES, D. D. M. Geoprocessamento Aplicado a Análise da Vulnerabilidade a Erosão na Bacia Hidrográfica do Rio Jaibaras - Ceara. 2011. Dissertação de Mestrado (Programa de Pós-Graduação em Geologia) Universidade Federal do Ceará, Fortaleza - CE.

PONZONI, F. J. SHIMABUKURO. Y.W. KUPLICH. T. M. Sensoriamento remoto da vegetação. 2 ed. São Paulo: Oficina de Textos. 2012.

RAMOS, P. R. S. DEUS, A. S. G, COSTA, S. O. S, GOMES, D. D. M. O sensoriamento remoto aplicado ao mapeamento, identificação e análise do uso do solo do município de Garanhuns - PE. Pernambuco. 2014.

RODRIGUES, E. L. FERNANDES, D. H. F. ELMIRO, M. A. T. FARIA, S. D.

Avaliação da cobertura vegetal por meio dos índices de vegetação SR, NDVI, SAVI e EVI na sub-bacia do Vale do Rio Itapecerica, Alto São Francisco, em Minas Gerais. Anais XVI Simpósio Brasileiro de Sensoriamento Remoto - SBSR, Foz do Iguaçu, PR. 2013. ROSA. R. Introdução ao sensoriamento remoto. 7 ed. São Paulo: UDUFU. 2009.

TRINCART, J. Ecodinâmica. Rio de Janeiro: FIBGE/SEPREN, 1977.

YI, X.S, LI, G.S. YIN, Y.Y. The impacts of grassland vegetation degradation on soil hydrologicaland ecological effects in the source region of the Yellow River--A casestudy in Junmuchang region of Maqin country. Procedia environmental sciences. 2012.

\section{AGRADECIMENTOS}

Os autores agradecem a Universidade de Pernambuco (UPE) pelo financiamento dos recursos dos projetos de pesquisa "Diagnóstico geoambiental das bacias hidrográficas do Estado de Pernambuco: geoprocessamento aplicado ao manejo e conservação dos recursos naturais", junto ao Programa de Fortalecimento Acadêmico (PFA/IC), e ao Laboratório de Geoprocessamento e Modelagem Ambiental pela cessão dos dados cartográficos vetorizados e imagens de satélites.

Recebido em: 14/08/2016

Aceito para publicação em: 01/10/2016 